\title{
Evaluation of the autoparametric pendulum vibration absorber for a Duffing system
}

\author{
Benjamın Vazquez-Gonzalez ${ }^{\mathrm{a}, *}$ and Gerardo Silva-Navarro ${ }^{\mathrm{b}}$ \\ ${ }^{a}$ Universidad Autonoma Metropolitana, Plantel Azcapotzalco, Departamento de Energia, Av. San Pablo No. 180, \\ Col. Reynosa Tamaulipas, C.P. 02200 Mexico, D.F. Mexico \\ ${ }^{\mathrm{b}}$ Centro de Investigacion y de Estudios Avanzados del I. P. N., Departamento de Ingenieria Electrica, Seccion de \\ Mecatronica, A.P. 14-740, C.P. 07360 Mexico, D.F. Mexico
}

Received 2007

Revised 2007

\begin{abstract}
In this work we study the frequency and dynamic response of a damped Duffing system attached to a parametrically excited pendulum vibration absorber. The multiple scales method is applied to get the autoparametric resonance conditions and the results are compared with a similar application of a pendulum absorber for a linear primary system. The approximate frequency analysis reveals that the nonlinear dynamics of the externally excited system are suppressed by the pendulum absorber and, under this condition, the primary Duffing system yields a time response almost equivalent to that obtained for a linear primary system, although the absorber frequency response is drastically modified and affected by the cubic stiffness, thus modifying the jumps defined by the fixed points. In the absorber frequency response can be appreciated a good absorption capability for certain ranges of nonlinear stiffness and the internal coupling is maintained by the existing damping between the pendulum and the primary system. Moreover, the stability of the coupled system is also affected by some extra fixed points introduced by the cubic stiffness, which is illustrated with several amplitude-force responses. Some numerical simulations of the approximate frequency responses and dynamic behavior are performed to show the steady-state and transient responses.
\end{abstract}

Keywords: Autoparametric systems, Duffing system, nonlinear frequency analysis, pendulum-type absorber

\section{Introduction}

Dynamic vibration absorbers are now classical devices, consisting of masses, springs and possibly dampers, which are configured and attached to a vibrating primary system to attenuate its undesirable forced dynamic response. The physical phenomenon governing the vibration attenuation involves the energy transfer from the primary system to the vibration absorbers. In most industrial applications, dynamic vibration absorbers have to interact with nonlinear mechanical systems, situation which certainly complicates the dynamic and frequency analysis. In fact, in some applications, a nonlinear vibration absorber may offer advantages, with respect to its linear counterpart, such as increased effective bandwidth and reduced resonant response. In Korenev and Reznikov [1], Sun et al. [2] and Harris [3] are presented collections of many vibration absorbers and their practical industrial applications.

Autoparametric systems are interesting vibrating systems, which consist of at least two nonlinearly coupled subsystems. The primary system can be externally excited by some harmonic force and, when it is connected to a secondary system (absorber) can be satisfied the so-called parametric excitation, that is, a mechanism that transfers the exogenous energy to the secondary system. In case the primary system is excited exactly or near its linear resonant frequency then, it is possible to get the principal parametric resonance for the secondary system (autoparametric

\footnotetext{
*Corresponding author. E-mail: bvg@correo.azc.uam.mx.
} 
interaction), attenuating the response of the primary system. See, e.g., Cartmell [4], Tondl et al. [5], Nayfeh and Mook [6] and references therein.

The autoparametric interaction between two mechanical systems can be encountered in many vibrating structures and machinery. This phenomenon has been widely discussed in the literature, since the work by Haxton and Barr [7]. Some pendulum-type absorbers have been applied for vibration protection on tower-pipes, chimneys, civil structures (buildings and bridges) affected by wind-induced and seismic vibrations, etc. (Korenev and Reznikov [1]).

The periodic and chaotic responses in the autoparametric pendulum vibration absorber for a linear mass-springdamper system have been studied, with the harmonic balance method, by Hatwal et al. [8]. In Cartmell et al. [9,10] is performed a nonlinear frequency analysis using the multiple scales method; here the two mode autoparametric interaction and robustness, against variations on the excitation frequency, are improved on the overall system by direct application of an on/off servomechanism, controlling the effective pendulum length and validating also the theoretical results in an experimental setup. A similar pendulum-type vibration absorber, with time delay in the internal feedback force, is used to illustrate the real-time application of a dynamic substructuring technique in Kyrichko et al. [11]. The harmonic balance method is also applied to study only the dynamic response of a pendulum vibration absorber in Song et al. [12]. Furthermore, Hsieh and Shaw [13] have analyzed the stability problem of inactive modes, parametrically driven by chaotic motions in the active mode, in certain configuration of a pendulum vibration absorber attached to a Duffing-van der Pol system. The analysis of a class of nonlinear dynamic vibration absorbers, with primary and secondary subsystems modelled by Duffing-van der Pol equations, is also considered by Zhu et al. [14] and references therein. It is important to remark that, in many cases, the nonlinear stiffness can arise from material nonlinearities as well as from geometrical nonlinearities, like in beams and plates [4-6,15].

This paper deals with an autoparametric pendulum vibration absorber connected to a damped Duffing primary system. The classical multiple scales method is then applied to get the autoparametric resonance conditions and the results are compared with similar applications of the pendulum absorber for linear primary systems in Cartmell et al. $[4,9,10]$. The approximate frequency analysis and amplitude-force responses allow us to conclude that, the nonlinear dynamics and the external harmonic force in the primary system are compensated by the pendulum vibration absorber and, therefore, the amplitude response is equivalent to that obtained for linear primary systems. In contrast, the nonlinearity certainly affects the frequency response of the pendulum absorber, with an enhancement of the effective bandwidth for the autoparametric interaction, phenomenon which may be related to the passive energy pumping described in detail by Jiang et al. [16] and references therein. The dynamic performance of the overall system is evaluated through numerical simulations.

\section{System description}

A damped Duffing primary system is attached to a pendulum vibration absorber (see Fig. 1), where the motion is restricted to a horizontal plane, thus neglecting the gravity effects. The primary system is a nonlinear vibrating system with mass $m_{1}$, a nonlinear spring with cubic stiffness function $k_{1} x+k_{2} x^{3}$ and linear viscous damping $c_{1}$. This mechanical system is also affected by an external harmonic force $F(t)=F_{0} \cos \Omega$ t, with amplitude $F_{0}$ and an excitation frequency $\Omega$ tuned at the principal parametric resonance associated to the primary system. For the purpose of attenuation of such harmonic vibrations is used a pendulum-type vibration absorber (secondary system), constituted by a lumped mass $m_{3}$ and a rigid bar with mass $m_{2}$ and moment of inertia $I_{2}$ with respect to its own center of mass. Both the primary and secondary subsystems are coupled by means of a linear spiral spring $k_{3}$ and linear viscous damping $c_{2}$. The lengths $l_{b}$ and $l$ denote the total pendulum length (bar) and the length measured along the bar from the position of the mass $m_{3}$ to the pivot point, respectively.

The equations of motion for the two degrees-of-freedom autoparametric vibration absorber, using Euler-Lagrange methodology, are obtained as follows (see also $[4,7,9]$ )

$$
\begin{aligned}
& \left(m_{1}+m_{2}+m_{3}\right) \ddot{x}+c_{1} \dot{x}+k_{1} x+k_{2} x^{3}-\left(\frac{1}{2} m_{2} l_{b}+m_{3} l\right)\left(\ddot{\theta} \sin \theta+\dot{\theta}^{2} \cos \theta\right)=F_{0} \cos \Omega t \\
& \left(\frac{1}{4} m_{2} l_{b}^{2}+I_{2}+m_{3} l^{2}\right) \ddot{\theta}+c_{2} \dot{\theta}+k_{3} \theta-\left(\frac{1}{2} m_{2} l_{b}+m_{3} l\right) \ddot{x} \sin \theta=0
\end{aligned}
$$




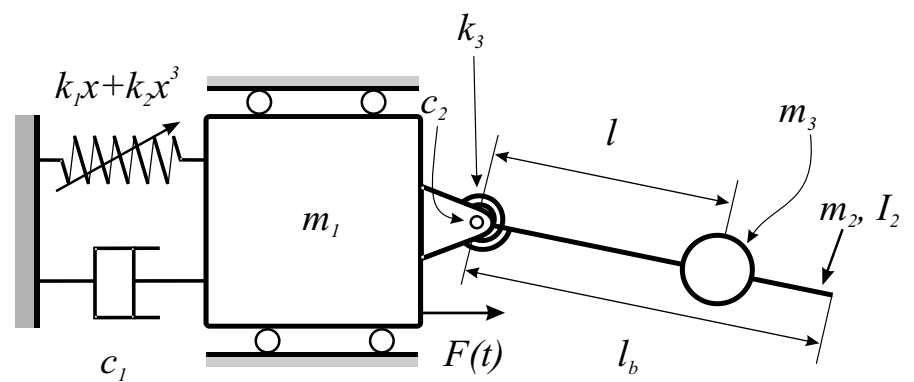

Fig. 1. Pendulum vibration absorber connected to a damped Duffing primary system.

where $x$ and $\theta$ denote the two generalized coordinates of the system, that is, the horizontal displacement of the primary system and the angular displacement of the pendulum, respectively. The external harmonic force is described by $F(t)=F_{0} \cos \Omega \mathrm{t}$, which directly affects the mass in the primary system $m_{1}$, with an excitation frequency close to the primary resonant frequency. Both Eqs (1) and (2) are internally coupled with nonlinear product terms, which is quite typical in autoparametric systems [4-6]. Note that, in absence of any autoparametric interaction (couplings), between both mechanical systems, the primary system motion can be transformed into a forced and damped Duffing vibrating system, with an inappropriate and large steady-state response because this will be operating under the external resonant condition described in $[4,9,10]$.

For small amplitudes in $x$ the influence of the cubic nonlinearity into the Duffing primary system Eq. (3) can be neglected because the dynamic behavior is predominantly linear, simplifying the frequency analysis to that already reported in $[4,9,10]$. However, for large motions in $x$ the cubic stiffness really affects the nonlinear dynamics in the primary system but, in this case, the inertial and centrifugal couplings with the pendulum absorber contribute to partially attenuate these undesirable effects.

By defining representative parameters and assuming small oscillations, one can transform the Eqs (1)-(2) in order to get an approximate analytical solution for the nonlinear frequency response. This procedure results in the following two coupled and nonlinear differential equations

$$
\begin{aligned}
& \ddot{x}+2 \varepsilon \zeta_{1} \omega_{1} \dot{x}+\omega_{1}^{2} x+\varepsilon \alpha x^{3}-\varepsilon h\left(\ddot{\theta} \theta+\dot{\theta}^{2}\right)=\varepsilon f \cos \Omega t \\
& \ddot{\theta}+2 \varepsilon \zeta_{2} \omega_{2} \dot{\theta}+\omega_{2}^{2} \theta-\varepsilon g \ddot{x} \theta=0
\end{aligned}
$$

where the system parameters are defined by

$$
\begin{aligned}
& M=m_{1}+m_{2}+m_{3}, \quad J=\frac{1}{4} m_{2} l_{b}^{2}+I_{2}+m_{3} l^{2}, \quad \omega_{1}^{2}=\frac{k_{1}}{M}, \quad \omega_{2}^{2}=\frac{k_{3}}{J}, \\
& \varepsilon \alpha=\frac{k_{2}}{M}, \quad \varepsilon \zeta_{1}=\xi_{1}=\frac{c_{1}}{2 \omega_{1} M}, \quad \varepsilon \zeta_{2}=\xi_{2}=\frac{c_{2}}{2 \omega_{2} J}, \quad \varepsilon f=\frac{F_{0}}{M}, \\
& \varepsilon h=\frac{1}{M}\left(\frac{1}{2} m_{2} l_{b}+m_{3} l\right), \quad \varepsilon g=\frac{1}{J}\left(\frac{1}{2} m_{3} l_{b}+m_{3} l\right),
\end{aligned}
$$

Here $\varepsilon$ represents a small perturbation parameter affecting the internal couplings between the pendulum absorber and the Duffing primary system, dampings, nonlinearities and external force into the system, whose influence is important in the present nonlinear frequency analysis. Specifically, the above perturbed equations include the cubic nonlinearity in the restoring force for the primary system, which is multiplied by the small perturbation parameter $\varepsilon$, that is, $\varepsilon \alpha$ with a constant $\alpha>0$ corresponding to a hardening spring. To establish the influence of the damping in the overall system, we have defined also the internal damping ratios as $\xi_{i}=\varepsilon \zeta_{i}, i=1,2$. In addition, the amplitude of the external harmonic force is perturbed as $\varepsilon f$. 


\subsection{Frequency analysis}

The classical method of multiple scales is used to find an approximate solution for the perturbed system Eqs (3)-(4) (see Cartmell [4], Nayfeh and Mook [6] and Awrejcewicz and Krysko [17]). Thus, the proposed perturbed solutions are expressed by

$$
\begin{aligned}
& x\left(T_{0}, T_{1}\right)=x_{0}\left(T_{0}, T_{1}\right)+\varepsilon x_{1}\left(T_{0}, T_{1}\right)+\ldots \\
& \theta\left(T_{0}, T_{1}\right)=\theta_{0}\left(T_{0}, T_{1}\right)+\varepsilon \theta_{1}\left(T_{0}, T_{1}\right)+\cdots
\end{aligned}
$$

where $T_{0}=t$ is the fast time scale, $T_{1}=\varepsilon t$ is the slow time scale and the remaining time scales are related by the perturbation as $T_{n}=\varepsilon^{n} t$, with $n=0,1,2, \ldots$ Time derivatives along different time scales lead to differential operators $d / d t=D_{0}+\varepsilon D_{1}+\ldots$ and $d^{2} / d t^{2}=D_{0}^{2}+2 \varepsilon D_{0} D_{1}+\ldots$. For approximation purposes we consider solutions Eqs (5)-(6) up to first order $\varepsilon$ terms.

The external and internal resonance conditions, describing the autoparametric interaction between the two-degreesof-freedom, are described by (see also [4-6])

$$
\begin{aligned}
& \Omega=\omega_{1}+\varepsilon \rho_{1} \\
& \omega_{1}=2 \omega_{2}+2 \varepsilon \rho_{2}
\end{aligned}
$$

where $\varepsilon \rho_{1}$ and $\varepsilon \rho_{2}$ define the external and internal detuning parameters, respectively.

Substitution of the proposed first order solutions Eqs (5)-(6) into Eqs (3)-(4) and grouping the zero and first order terms in $\varepsilon$, yields the following set of partial differential equations

$$
\begin{aligned}
& \varepsilon^{0}: D_{0}^{2} x_{0}+\omega_{1}^{2} x_{0}=0 \\
& \varepsilon^{1}: D_{0}^{2} x_{1}+\omega_{1}^{2} x_{1}=-2 \zeta_{1} \omega_{1} D_{0} x_{0}-2 D_{0} D_{1} x_{0}-\alpha x_{0}^{3}+h\left(D_{0} \theta_{0}\right)^{2}+h\left(D_{0}^{2} \theta_{0}\right) \theta_{0}+f \cos \left(\Omega T_{0}\right) \\
& \varepsilon^{0}: D_{0}^{2} \theta_{0}+\omega_{2}^{2} \theta_{0}=0 \\
& \varepsilon^{1}: D_{0}^{2} \theta_{1}+\omega_{2}^{2} \theta_{1}=g\left(D_{0}^{2} x_{0}\right) \theta_{0}-D_{1} D_{0} \theta_{0}-2 \zeta_{2} \omega_{2} D_{0} \theta_{0}
\end{aligned}
$$

The proposed solutions for Eqs (9) and (11) are harmonics expressed in their polar forms as follows

$$
\begin{aligned}
& x_{0}=A\left(T_{1}\right) e^{i \omega_{1} T_{0}}+\bar{A}\left(T_{1}\right) e^{-i \omega_{1} T_{0}} \\
& \theta_{0}=B\left(T_{1}\right) e^{i \omega_{2} T_{0}}+\bar{B}\left(T_{1}\right) e^{-i \omega_{2} T_{0}}
\end{aligned}
$$

The solutions for Eqs (10) and (12) strongly depend on the above solutions for Eqs (9) and (11). Hence, substituting Eqs (13)-(14) into Eqs (10) and (12) results in

$$
\begin{aligned}
D_{0}^{2} x_{1}+\omega_{1}^{2} x_{1}= & -2 i \zeta_{1} \omega_{1}^{2} A e^{i \omega_{1} T_{0}}-2 i \omega_{1} A^{\prime} e^{i \omega_{1} T_{0}}-\alpha A^{3} e^{3 i \omega_{1} T_{0}}-3 \alpha A^{2} \bar{A} e^{i \omega_{1} T_{0}}-2 h \omega_{2} B^{2} e^{2 i \omega_{2} T_{0}} \\
& +\frac{1}{2} f e^{i \Omega T_{0}}+C . C . \\
D_{0}^{2} \theta_{1}+\omega_{2}^{2} \theta_{1}= & -g \omega_{1}^{2} A B e^{i\left(\omega_{1}+\omega_{2}\right) T_{0}}-g \omega_{1}^{2} A \bar{B} e^{i\left(\omega_{1}-\omega_{2}\right) T_{0}}-2 i \omega_{2} B^{\prime} e^{i \omega_{2} T_{0}}-2 i \zeta_{2} \omega_{2} B e^{i \omega_{2} T_{0}}+C . C .
\end{aligned}
$$

where C.C. stands for complex conjugate terms. Cancellation of secular terms from Eqs (15) and (16), and using the polar forms $A\left(T_{1}\right)=\frac{1}{2} a\left(T_{1}\right) e^{i \delta\left(T_{1}\right)}$ and $B\left(T_{1}\right)=\frac{1}{2} b\left(T_{1}\right) e^{i \gamma\left(T_{1}\right)}$, leads to the following equations described on the slow time scale $T_{1}$

$$
\begin{aligned}
& -i \zeta_{1} \omega_{1}^{2} a-i \omega_{1} a^{\prime}+\omega_{1} a \delta^{\prime}-\frac{3}{8} \alpha a^{3}-\frac{1}{2} h \omega_{2}^{2} b^{2} e^{i \phi_{1}}+\frac{1}{2} f e^{i \phi_{2}}=0 \\
& -\frac{1}{4} g \omega_{1}^{2} a b e^{-i \phi_{1}}-i \omega_{2} b^{\prime}+b \omega_{2} \gamma^{\prime}-i b \zeta_{2} \omega_{2}^{2}=0 \\
& \phi_{1}=\rho_{1} T_{1}-\delta \\
& \phi_{2}=2 \gamma-\delta-2 \rho_{2} T_{1}
\end{aligned}
$$

where $a^{\prime}, b^{\prime}, \delta^{\prime}$ and $\gamma^{\prime}$ denote differentiation with respect to the slow time scale $T_{1}$. 


\subsection{Steady-state response}

The steady state responses of the overall system are computed for $a^{\prime}=0, b^{\prime}=0, \delta^{\prime}=\rho_{1}$ and $\gamma^{\prime}=\frac{\rho_{1}}{2}+\rho_{2}$. The amplitude responses are obtained by taking real and imaginary parts in Eqs (17)-(20) for the steady-state conditions, resulting

$$
\begin{aligned}
& \omega_{1} a \rho_{1}-\frac{3}{8} \alpha a^{3}-\frac{1}{2} h \omega_{2}^{2} b^{2} \cos \phi_{1}+\frac{1}{2} f \cos \phi_{2}=0 \\
& -\zeta_{1} \omega_{1}^{2} a-\frac{1}{2} h \omega_{2}^{2} b^{2} \sin \phi_{1}+\frac{1}{2} f \sin \phi_{2}=0 \\
& -\frac{1}{4} g \omega_{1}^{2} a b \cos \phi_{1}+b \omega_{2}\left(\frac{\rho_{1}}{2}+\rho_{2}\right)=0 \\
& \frac{1}{4} g \omega_{1}^{2} a b \sin \phi_{1}-b \zeta_{2} \omega_{2}^{2}=0
\end{aligned}
$$

Now, by solving the above equations we can get the approximate amplitude responses for the primary and secondary subsystems. Specifically, for the primary system is obtained that

$$
a=\frac{4 \omega_{2}^{2}}{(\varepsilon g) \omega_{1}^{2}} \sqrt{\left(\frac{\Omega}{2 \omega_{2}}-1\right)^{2}+\left(\varepsilon \zeta_{2}\right)^{2}}
$$

The approximate amplitude response for the secondary system is described implicitly in terms of the real solutions for the algebraic equation

$$
b^{4}+Q b^{2}+R=0
$$

where

$$
\begin{aligned}
Q= & \frac{12 \omega_{2}(\varepsilon \alpha)\left(\Omega-2 \omega_{2}\right)^{3}}{(\varepsilon h)(\varepsilon g)^{3} \omega_{1}^{6}}+\frac{48 \omega_{2}^{3}(\varepsilon \alpha)\left(\varepsilon \zeta_{2}\right)^{2}\left(\Omega-2 \omega_{2}\right)}{(\varepsilon h)(\varepsilon g)^{3} \omega_{1}^{6}}-\frac{8\left(\Omega-2 \omega_{2}\right)\left(\Omega-\omega_{1}\right)}{(\varepsilon h)(\varepsilon g) \omega_{1} \omega_{2}}+\frac{16\left(\varepsilon \zeta_{1}\right)\left(\varepsilon \zeta_{2}\right)}{(\varepsilon g)(\varepsilon h)} \\
R= & \frac{36 \omega_{2}^{2}(\varepsilon \alpha)^{2}}{(\varepsilon h)^{2}(\varepsilon g)^{3} \omega_{1}^{6}}\left[\left(\frac{\Omega}{2 \omega_{2}}-1\right)^{2}+\left(\varepsilon \zeta_{2}\right)^{2}\right]^{\frac{3}{2}}-\frac{768 \omega_{2}^{4}(\varepsilon \alpha)\left(\Omega-\omega_{1}\right)}{(\varepsilon h)^{2}(\varepsilon g)^{4} \omega_{1}^{7}}\left[\left(\frac{\Omega}{2 \omega_{2}}-1\right)^{2}+\left(\varepsilon \zeta_{2}\right)^{2}\right]^{2} \\
& +\frac{64\left[\left(\Omega-\omega_{1}\right)^{2}+\omega_{1}^{2}\left(\varepsilon \zeta_{1}\right)^{2}\right]}{(\varepsilon h)^{2}(\varepsilon g)^{2} \omega_{2}^{2}}\left[\left(\frac{\Omega}{2 \omega_{2}}-1\right)^{2}+\left(\varepsilon \zeta_{2}\right)^{2}\right]-\frac{(\varepsilon f)^{2}}{(\varepsilon h)^{2} \omega_{2}^{4}}
\end{aligned}
$$

Note that, the primary system response Eq. (25) does not depend on the cubic stiffness and the external force into the damped Duffing system. In fact, this amplitude is the same as that determined by Cartmell et al. [4,9,10], where there are only linear elements in the primary system. In contrast, the secondary system response in Eq. (26) is indeed influenced by the cubic nonlinearity $\varepsilon \alpha$, precisely in the first two terms of the parameters $Q$ and $R$, meaning that the cubic term has been transferred from the primary system to the pendulum absorber. Moreover, when the cubic stiffness is neglected $(\varepsilon \alpha=0)$, the system response coincides also with that reported by Cartmell et al. [4,9]. Therefore, one can conclude some kind of robustness of the pendulum vibration absorber against the cubic stiffness and the external force, both affecting the primary system.

Remark: A similar multiple scales analysis can prove that, there is also an approximate analytical solution for the overall system Eqs (3)-(4) when the pendulum absorber remains with any oscillation or is locked up at $\theta(t) \equiv 0$. This case clearly corresponds to the solution of a single degree-of-freedom system, for the well-known damped Duffing system Eq. (3), operating under the external resonance condition. The nonlinear frequency response is obtained by replacing $b=0$ in Eqs (21)-(22) and, after some algebraic manipulations, results the following equation

$$
\frac{9}{16}(\varepsilon \alpha)^{2} a^{6}-3(\varepsilon \alpha) \omega_{1}\left(\varepsilon \rho_{1}\right) a^{4}+4 \omega_{1}^{2}\left[\left(\varepsilon \rho_{1}\right)^{2}+\omega_{1}^{2}\left(\varepsilon \zeta_{1}\right)^{2}\right] a^{2}-(\varepsilon f)^{2}=0
$$


Note that, for primary linear systems $(\varepsilon \alpha=0)$ the above equation is simplified to a simple quadratic equation, whose solution is equivalent to that reported by Cartmell and Lawson [9]. This linear solution is given by

$$
a=\frac{\varepsilon f}{2 \omega_{1} \sqrt{\left(\varepsilon \rho_{1}\right)^{2}+\omega_{1}^{2}\left(\varepsilon \zeta_{1}\right)^{2}}}
$$

\subsubsection{Fixed points}

By taking the real and imaginary parts in Eqs (17)-(20) and solving them for $a^{\prime}, b^{\prime}, \phi^{\prime}{ }_{1}$ and $\phi_{2}^{\prime}$ result the differential equations

$$
\begin{aligned}
a^{\prime} & =\frac{-\zeta_{1} \omega_{1}^{2} a-\frac{1}{2} h \omega_{2}^{2} b^{2} \sin \phi_{1}+\frac{1}{2} f \sin \phi_{2}}{\omega_{1}} \\
b^{\prime} & =\frac{\frac{1}{4} g \omega_{1}^{2} a b \sin \phi_{1}-\zeta_{2} \omega_{2}^{2} b}{\omega_{2}} \\
\phi_{1}^{\prime} & =\frac{\omega_{1} a \rho_{1}-\frac{3}{8} \alpha a^{3}-\frac{1}{2} h \omega_{2}^{2} b^{2} \cos \phi_{1}+\frac{1}{2} f \cos \phi_{2}}{\omega_{1} a} \\
\phi_{2}^{\prime} & =\frac{\frac{1}{2} g \omega_{1}^{3} a^{2} \cos \phi_{1}-\frac{3}{8} \omega_{2} \alpha a^{3}-\frac{1}{2} h \omega_{2}^{3} b^{2} \cos \phi_{1}+\frac{1}{2} \omega_{2} f \cos \phi_{2}+\omega_{1} \omega_{2} a 2 \rho_{2}}{\omega_{1} \omega_{2} a}
\end{aligned}
$$

Now, solving the system Eqs (29)-(32) for $a^{\prime}=0, b^{\prime}=0, \phi_{1}^{\prime}=0$ and $\phi_{2}^{\prime}=0$ yields the fixed points. In this case, the fixed points for the exact tunings $\rho_{1}=\rho_{2}=0\left(\omega_{1}=\Omega\right.$ and $\left.\omega_{2}=\omega_{1} / 2\right)$ are calculated as follows

$$
a= \pm \frac{\zeta_{2}}{g}, \quad b=\frac{u}{g \omega_{1}^{3} \omega_{2}}, \quad \phi_{1}= \pm \frac{\pi}{2}, \quad \phi_{2}=\arctan \left[ \pm \frac{(s+q)}{-r}\right]
$$

where

$$
r=\frac{48 \alpha \zeta_{2}^{3} \omega_{2}^{6}}{g^{3} \omega_{1}^{6} f}, \quad s=8 \zeta_{1} \zeta_{2} \omega_{1}^{6} \omega_{2}^{2} g, \quad q=\frac{h u^{2}}{g^{3} \omega_{1}^{6} f}, \quad p=2304 \zeta_{2}^{6} \omega_{2}^{12} \alpha^{2}+64 \zeta_{2}^{2} \zeta_{1}^{2} g^{4} \omega_{1}^{12} \omega_{2}^{4}-f^{2} g^{6} \omega_{1}^{12}
$$

Here the amplitudes $b$ and $q$ are parameterized in terms of the parameter $u$, which represents the roots of the polynomial

$$
\left(h^{2} g^{2}\right) u^{4}-\left(16 \zeta_{2} \zeta_{1} h \omega_{1}^{6} \omega_{2}^{2} g^{3}\right) u^{2}+p=0
$$

Therefore, $b$ and $q$ are directly affected by the parameters $\alpha$ and $f$ associated to the cubic stiffness and the external harmonic force, respectively. The total number of real fixed points, including the trivial solution, is at least ten. In fact, the cubic stiffness adds three more non-trivial fixed points to the case studied by Cartmell and Lawson [9].

\section{Simulation results}

To evaluate the dynamic and steady-state performance of the damped primary Duffing system connected to the pendulum vibration absorber, we consider the model Eqs (1)-(2) and the physical system parameters given in Table 1. It is important to remark that, in this model Eqs (1)-(2) is not supposed the small angles assumption, in contrast to the perturbed model Eqs (3)-(4) used to get the approximate frequency analysis. However, the dynamic responses and steady-states behavior are quite consistent.

Some numerical simulations are illustrated for variations on the external harmonic force, for the exact tuning as well as for some non-exact tunings. For the pendulum absorber is performed a comparison of different frequency responses obtained for two different amplitudes in the external harmonic force. The approximate frequency and dynamic responses are obtained using Maple ${ }^{\circledR} 9.5$ and Simnon ${ }^{\circledR} 3.0$, respectively.

First of all, the amplitude frequency response for the damped Duffing primary system is shown in Fig. 2. This results when $F_{0}=10 N$ and there is no autoparametric interaction with the pendulum absorber, that is, when $\theta(t) \equiv$ 
Table 1

Physical system parameters

\begin{tabular}{lll}
\hline$m_{1}=5.0 \mathrm{~kg}$ & $m_{2}=1.0 \mathrm{~kg}$ & $m_{3}=1 \mathrm{~kg}$ \\
$k_{1}=474.92 \mathrm{~N} / \mathrm{m}$ & $k_{2}=77.0 \mathrm{~N} / \mathrm{m}^{3}$ & $k_{3}=4.0 \mathrm{~N}-\mathrm{m} / \mathrm{rad}$ \\
$c_{1}=1.0 \mathrm{~N} /(\mathrm{m} / \mathrm{s})$ & $c_{2}=0.01 \mathrm{~N}-\mathrm{m} /(\mathrm{rad} / \mathrm{s})$ & $F_{0}=10 \mathrm{~N}$ \\
$l=0.3 \mathrm{~m}$ & $l_{b}=0.5 \mathrm{~m}$ & \\
$\omega_{1}=8.2368 \mathrm{rad} / \mathrm{s}$ & $\omega_{2}=4.1184 \mathrm{rad} / \mathrm{s}$ & $\varepsilon \zeta_{1}=0.00867$ \\
$\varepsilon \zeta_{2}=0.00515$ & $\varepsilon \alpha=74.25\left(\mathrm{~N} / \mathrm{m}^{3}\right) / \mathrm{kg}$ & $\varepsilon h=0.7854 \mathrm{~m}$ \\
$\varepsilon g=2.3321 \mathrm{~m}^{-1}$ & $\varepsilon f=1.4285 \mathrm{~m} / \mathrm{s}^{2}$ & $\varepsilon=0.55 \mathrm{~kg} \cdot \mathrm{m}$ \\
\hline
\end{tabular}

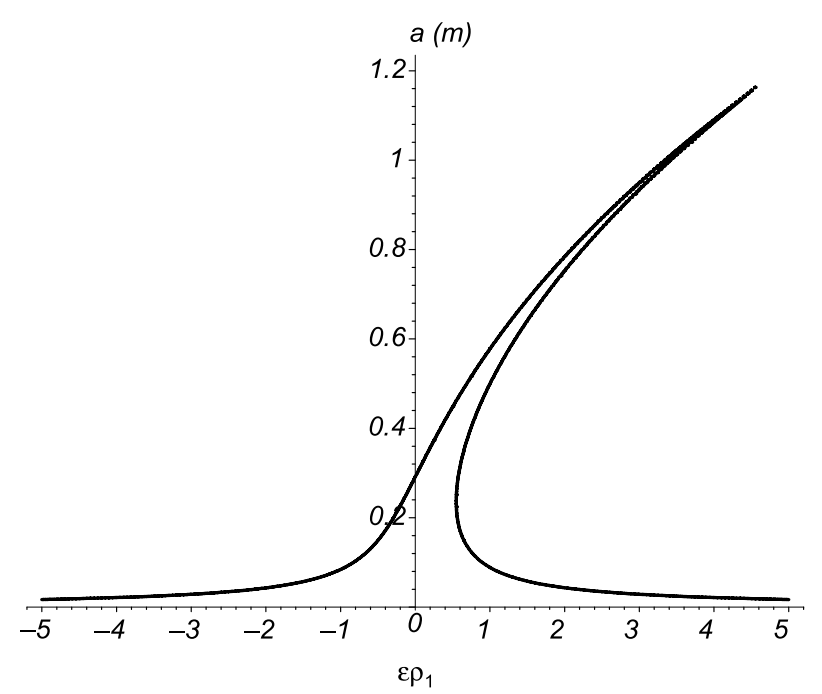

Fig. 2. Frequency response for the damped Duffing system when $F_{0}=10 \mathrm{~N}$ and the pendulum absorber is locked up at $\theta(t) \equiv 0$.

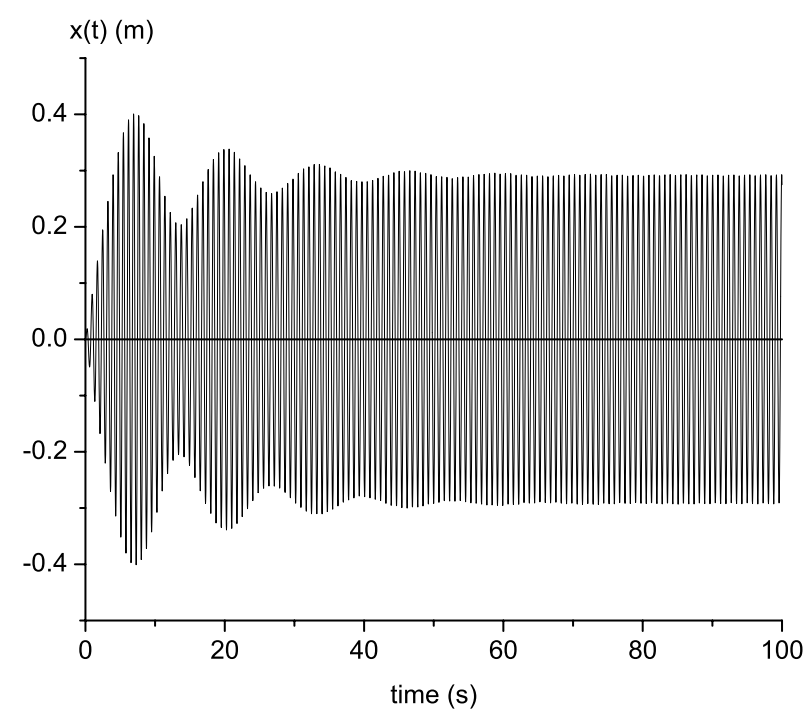

Fig. 3. Dynamic behavior of the damped Duffing system when $F_{0}=10 \mathrm{~N}$ and there is no autoparametric interaction $(\theta(t) \equiv 0)$.

0 (e.g., using zero initial conditions). This frequency response clearly corresponds to the well-known behavior for a damped Duffing system, describing the classical bent response due to the cubic stiffness associated to a hardening spring and when no energy pumping occurs. The corresponding dynamic behavior for the primary system, under the external resonance condition $\left(\varepsilon \rho_{1}=0\right)$, is described in Fig. 3. Note that the steady-state response achieves a 


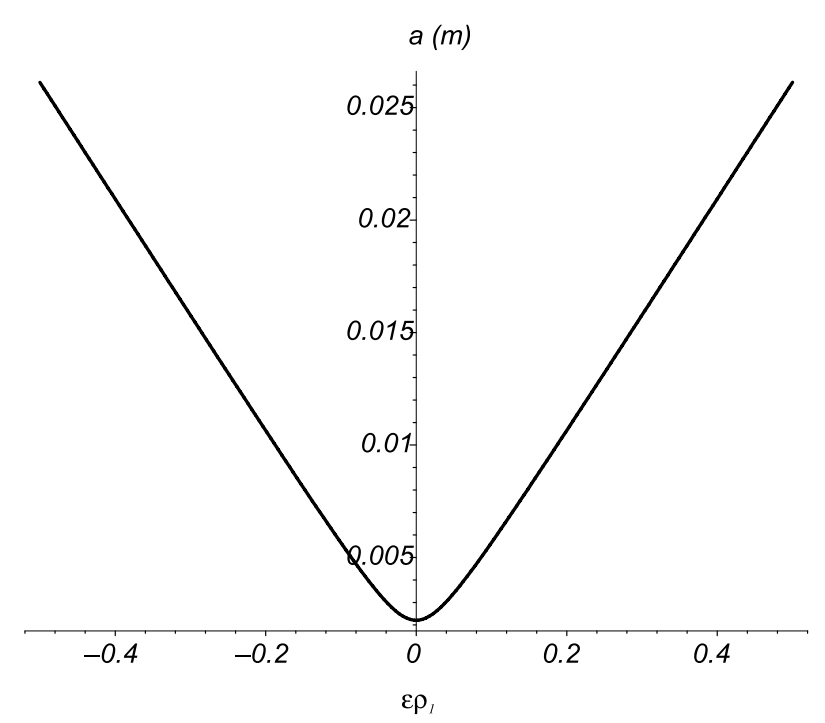

Fig. 4. Frequency response for the primary system when $F_{0}=10 \mathrm{~N}$.

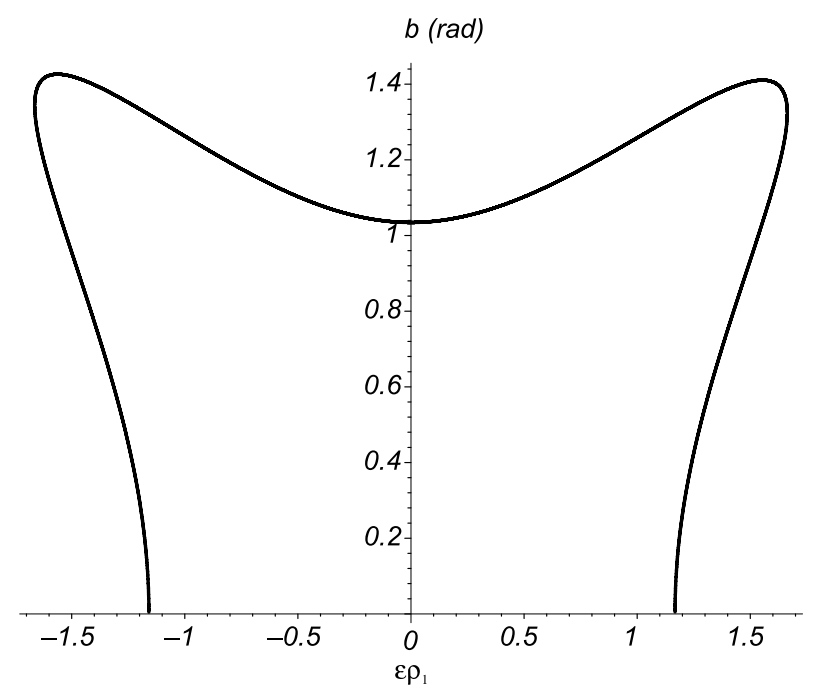

Fig. 5. Frequency response for the secondary system when $F_{0}=10 \mathrm{~N}$

large value ( $a=0.3$ ), although this is bounded by the existing damping.

The frequency responses for both the primary and secondary systems, under autoparametric interaction and external harmonic force with amplitude $F_{0}=10 \mathrm{~N}$, are described in Figs 4 and 5, respectively. These responses are obtained directly from Eqs (25) and (26). Note that, exactly at the tuning condition $\varepsilon \rho_{1}=0$ and also in a small neighborhood, there is a reasonable energy pumping from the primary system to the pendulum absorber. In fact, for the region delimited by detunings $\left|\varepsilon \rho_{1}\right| \leqslant 1.55$ (Fig. 5), it is clearly appreciated how the influence of the cubic stiffness (hardening spring) in the primary system is transferred to the pendulum absorber, situation significantly distinctive with respect to those results in Cartmell and Lawson [9]. The transient responses of the overall system for exact tuning $\left(\varepsilon \rho_{1}=0\right)$ and initial conditions leading to autoparametric interaction (i.e., $x(0)=0 \mathrm{~m}, \dot{x}(0)=0 \mathrm{~m} / \mathrm{s}, \theta(0)=0.6 \mathrm{rad}, \dot{\theta}(0)=0 \mathrm{rad} / \mathrm{s})$ are illustrated in Figs 6 and 7 . Here one can observe the attenuation on the primary Duffing system response with respect to the motion of the pendulum absorber. This dynamic behavior is quite consistent with the frequency responses predicted in Figs 4 and 5. 


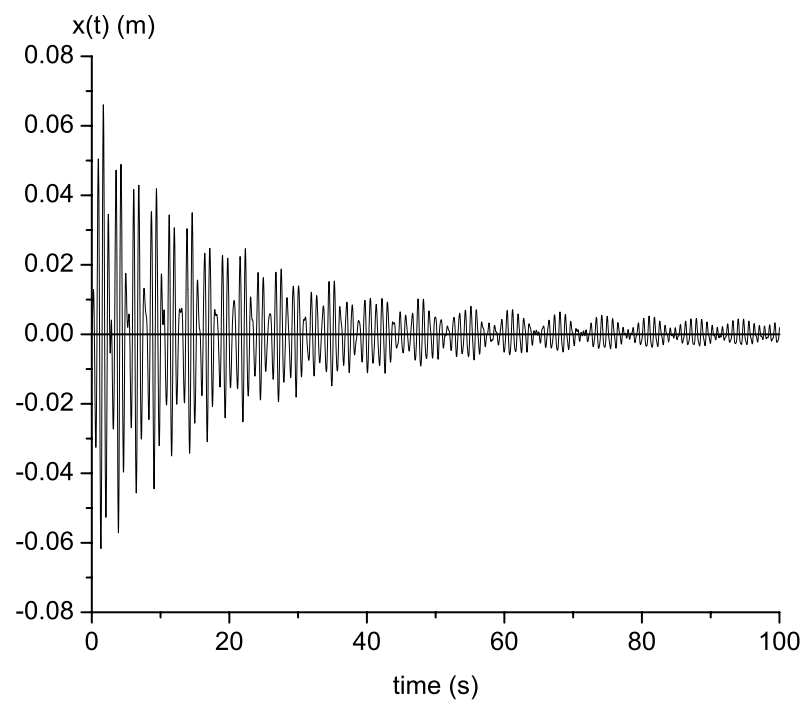

Fig. 6. Dynamic behavior for the primary system when $\varepsilon \rho_{1}=0$ and $F_{0}=10 \mathrm{~N}$.

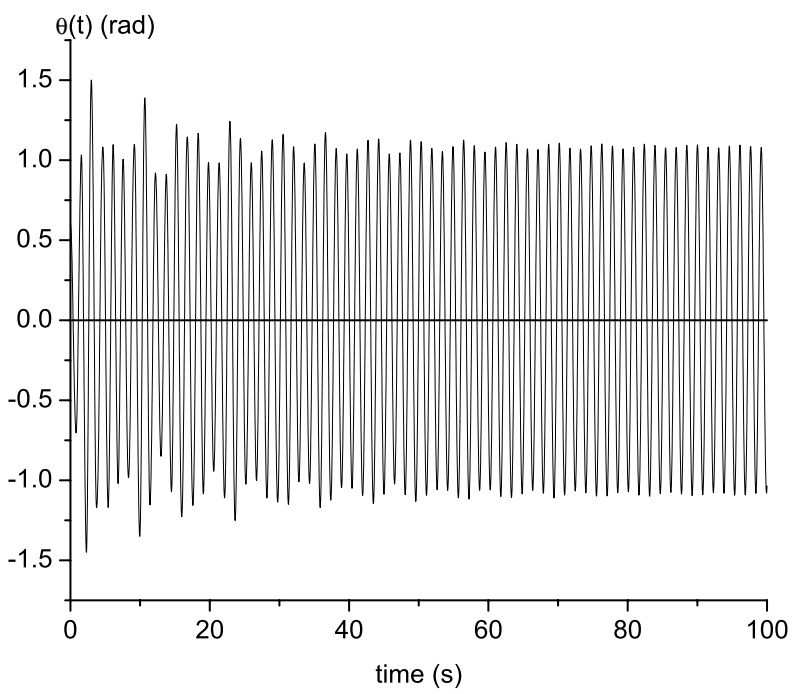

Fig. 7. Dynamic behavior for the secondary system when $\varepsilon \rho_{1}=0$ and $F_{0}=10 N$.

The frequency responses in Figs 4 and 5 exhibit two different steady-state behaviors, for the exact resonance condition $\varepsilon \rho_{1}=0$ with $(a=0.0022, b=1.04)$ and increasing values up to $\left|\varepsilon \rho_{1}\right| \approx 1.55$ yield $(a=0.08, b=1.41)$. Detunings $\left|\varepsilon \rho_{1}\right|>1.55$ suddenly reduce the absorption property.

The amplitude-force responses for the pendulum absorber when $\varepsilon \rho_{1}=0$ (tuned) and $\varepsilon \rho_{1}=1.55$ (detuned) are shown in Figs 8 and 9, respectively. In the latter case a smaller frequency response is identified for small amplitudes in the external harmonic force (see also Fig. 5). For instance, we observe in Fig. 8 that, for the parametric force $\varepsilon f=1.4286\left(F_{0}=10 \mathrm{~N}\right)$ the amplitude of the absorber is approximately $b=1.04$, which is also verified in Fig. 5. Another interesting condition is identified by the detuning $\varepsilon \rho_{1}=1.55$, whose amplitude-force response is shown in Fig. 9; here one can observe the presence of a region denoted by $b^{*}$, corresponding to values of the force that do not satisfy the absorption property. In other words, when the system is detuned and $\varepsilon f<0.15$, the solution is too small to induce any motion on the pendulum absorber and, as a consequence, the absorption property on the primary Duffing system can not be achieved. This amplitude-force response exhibits two branches defined by the solutions of Eq. (26), such that the solution $b^{*}$ corresponds to values of the force $\varepsilon f$ that do not satisfy the lock conditions 


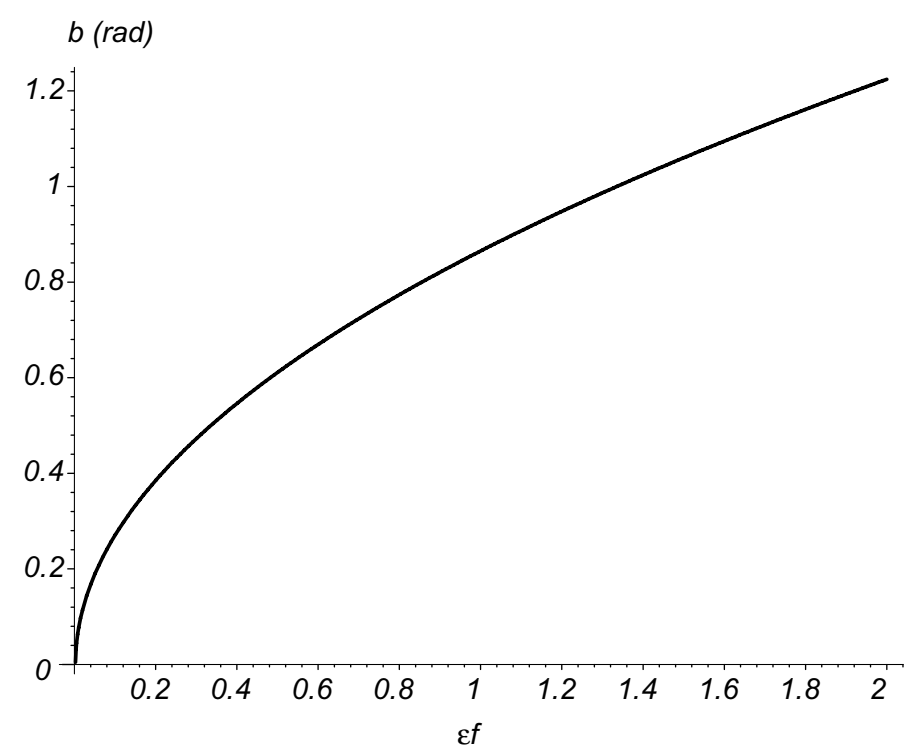

Fig. 8. Amplitude-force response for the absorber when $\varepsilon \rho_{1}=0$.

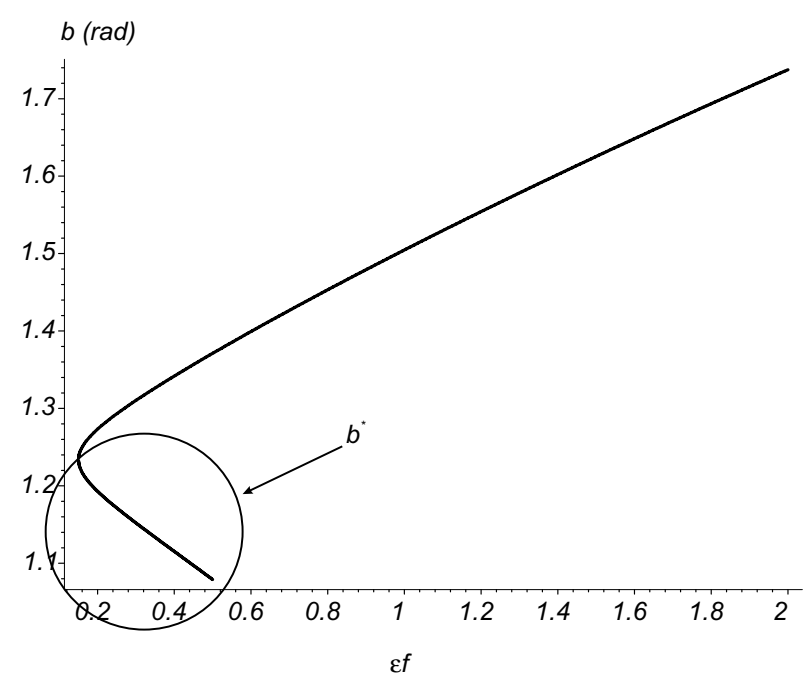

Fig. 9. Amplitude-force response for the absorber when when $\varepsilon \rho_{1}=1.55$.

(autoparametric interaction), which is rather equivalent to a force threshold leading to the absorption condition in the primary system.

In order to illustrate the sensitiveness of the overall system to variations on the amplitude of the external harmonic force, we now study the effect of varying the magnitude of the external force on the frequency responses. To do this, we compute the amplitude-force responses when $\varepsilon f=0.2857\left(F_{0}=2 \mathrm{~N}\right)$ and exact tuning $\left(\varepsilon \rho_{1}=0\right)$. The frequency response for the pendulum absorber is depicted in Fig. 10, while the frequency response for the primary Duffing system is identical to that in Fig. 4 (see also Eq. (25)). In this context, it results evident the absorption mechanism of the cubic stiffness $\varepsilon \alpha$ and the external force $\varepsilon f$ both directly affecting the primary system, which are actually transferred to the pendulum absorber. The corresponding dynamic behavior is shown in Figs 11 and 12 , whose steady-state amplitudes are also compatible with their frequency responses $(a=0.0022, b=0.461)$. Moreover, the region of autoparametric interaction is simultaneously influenced by the detuning parameter $\varepsilon \rho_{1}$ and 


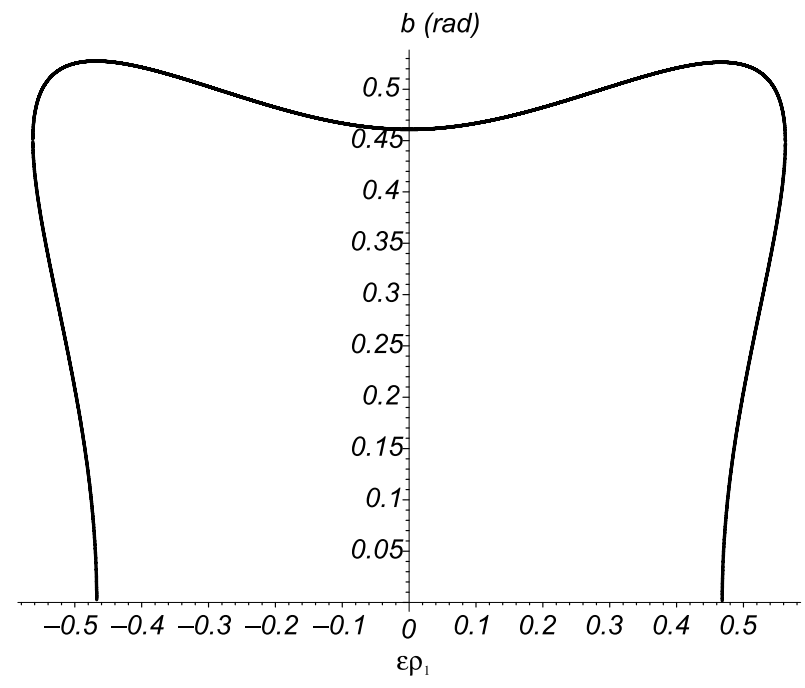

Fig. 10. Absorber frequency response when $F_{0}=2 \mathrm{~N}$.

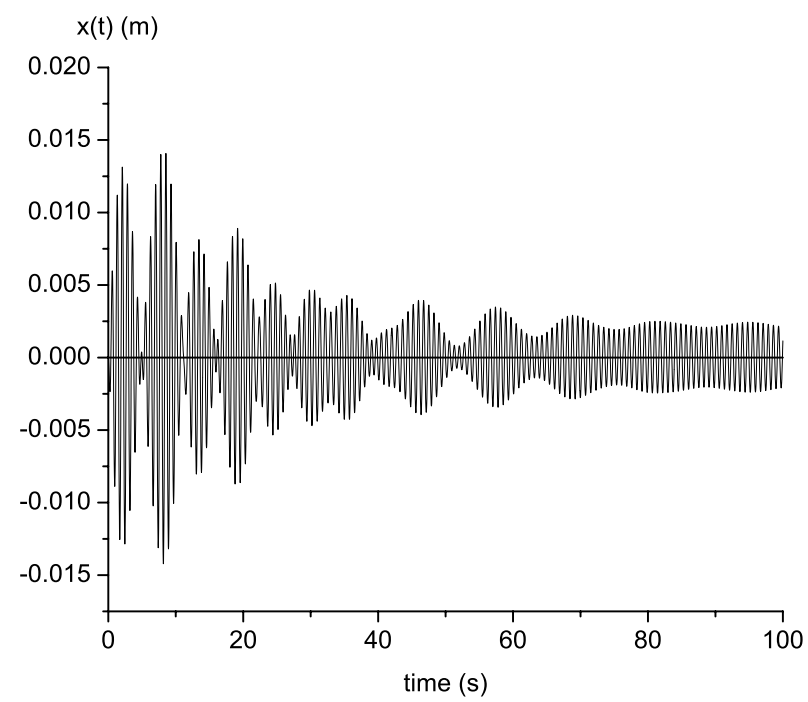

Fig. 11. Dynamic behavior of the primary system when $\varepsilon \rho_{1}=0$ and $F_{0}=2 \mathrm{~N}$.

the parametric force $\varepsilon f$ (see Figs 8 and 9) and, therefore, the effective absorption in the overall system can be maintained in spite of variations on the excitation frequency and the amplitude force.

\subsection{Stability analysis}

In general, the steady-state absorption is affected by the parametric force $\varepsilon f$, the cubic stiffness $\varepsilon \alpha$, the detuning $\varepsilon \rho_{1}$ and the initial conditions, which modify the phases and synchronization between the primary system and the pendulum absorber. Some specific operating region is encountered when $\left|\varepsilon \rho_{1}\right| \leqslant 1.55$, where the absorption is guaranteed and the amplitude response of the absorber is minimal (see, e.g., Figs 5 and 10). It is important to remark that, the amplitude response for the primary system $a$ is independent from the parametric force $\varepsilon f$ and the cubic stiffness (see Eq. (25) and Fig. 4).

From Eqs (29)-(32) the fixed points are parameterized by the detuning parameter $\varepsilon \rho_{1}$, where complicated expressions define their numerical values. The stability of such fixed points is analyzed by means of the Routh- 


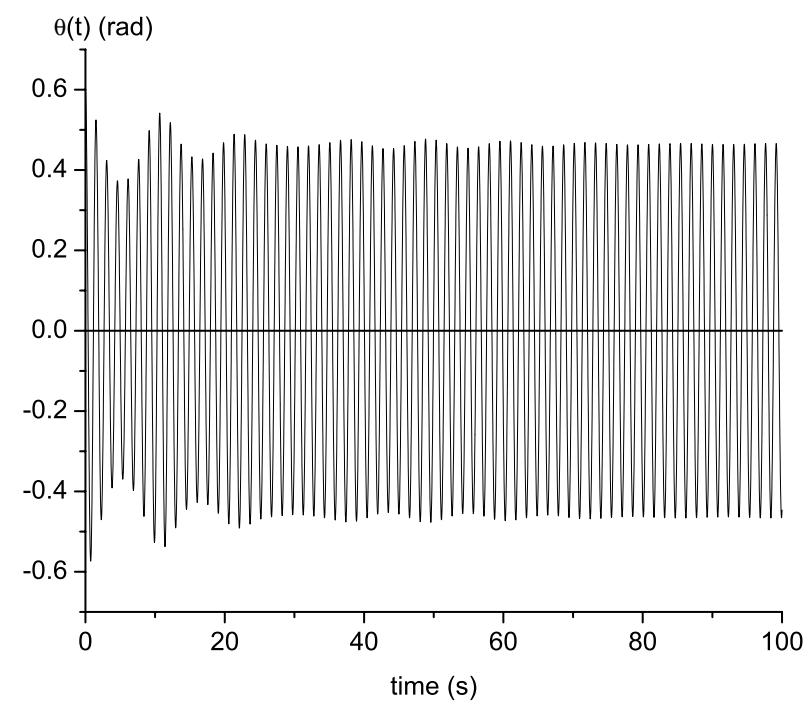

Fig. 12. Dynamic behavior of the pendulum absorber when $\varepsilon \rho_{1}=0$ and $F_{0}=2 \mathrm{~N}$.

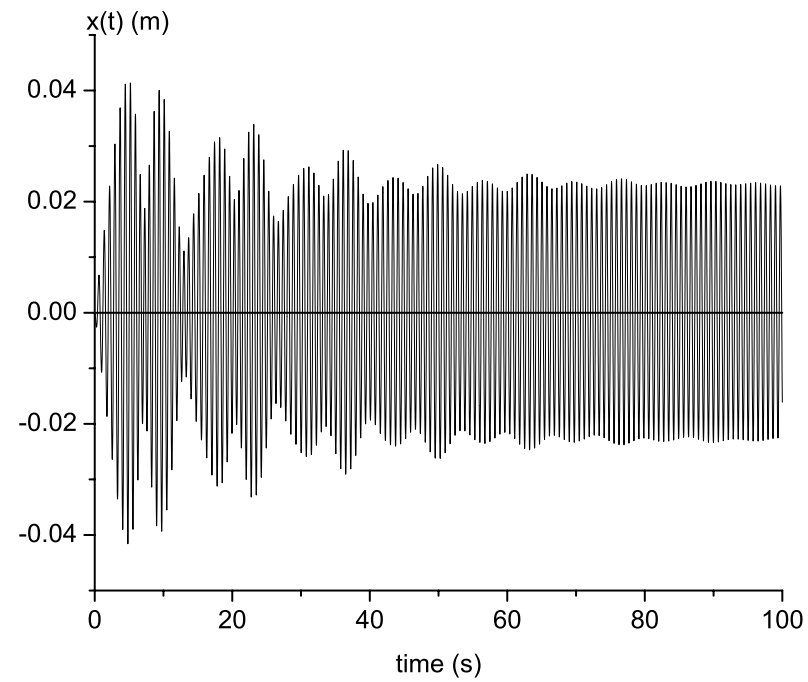

Fig. 13. Dynamic response for the damped primary Duffing system when $\varepsilon \rho_{1}=0.46$ and $F_{0}=2 \mathrm{~N}$.

Hurwitz criteria. For instance, when $\varepsilon \rho_{1}=0.46$ and $\varepsilon f=0.2857\left(F_{0}=2 \mathrm{~N}\right)$ the fixed point $(a=0.024, b=$ $0.527, \phi_{1}=0.0919, \phi_{2}=0.2495$ ) is unstable. In this case, the transient responses for the primary Duffing system and pendulum absorber are shown in Figs 13 and 14, respectively.

\section{Conclusions}

The dynamic response and nonlinear frequency analysis of a damped Duffing system attached to a pendulum vibration absorber, both operating under the external and internal resonance conditions, are discussed. In particular, the effects introduced by the cubic stiffness and the external harmonic force, into the dynamics and frequency response of the primary system, are certainly transferred to the pendulum-type absorber and, hence, the overall system performance enjoys some robustness against nonlinearities, external forces as well as variations on the excitation frequency. The analysis reveals that, when the magnitude of the external force is below a small force 


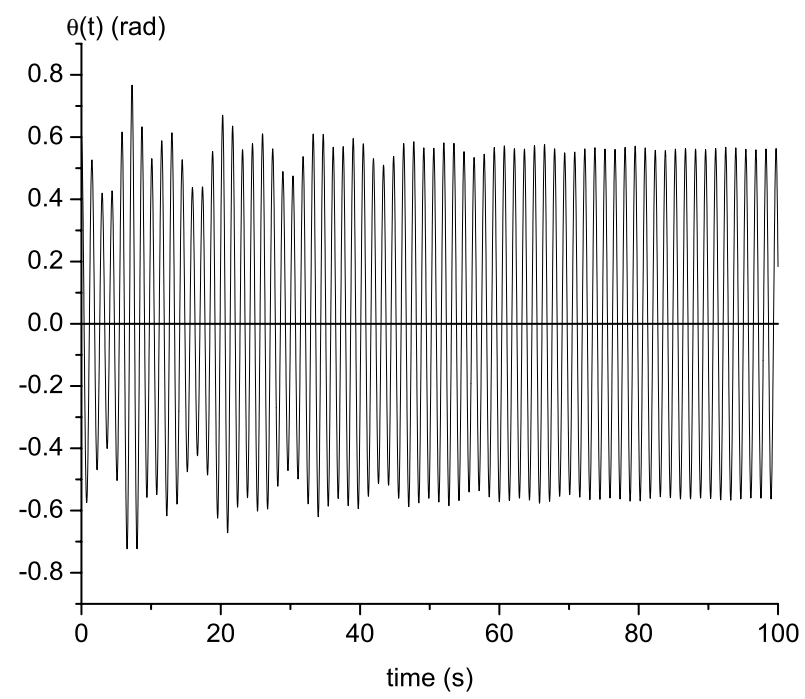

Fig. 14. Dynamic response for the pendulum absorber when $\varepsilon \rho_{1}=0.46$ and $F_{0}=2 \mathrm{~N}$.

threshold, then, there is not sufficient exogenous energy to bring on the system to the autoparametric interaction and, as a consequence, the vibration attenuation. Moreover, for excitation frequencies close to the exact tuning is still possible to reduce simultaneously the amplitude responses of the primary and secondary systems. This operating condition is possible due to the presence of the hardening spring and an appropriate force that excite the overall system to work preserving the stability properties.

\section{Acknowledgement}

The authors would like to acknowledge the useful and fruitful comments of the anonymous reviewers.

\section{References}

[1] B.G. Korenev and L.M. Reznikov, Dynamic Vibration Absorbers: Theory and Technical Applications, London: John Wiley \& Sons, 1993.

[2] J.Q. Sun, M.R. Jolly and M.A. Norris, Passive, adaptive and active tuned vibration absorbers - a survey, Transactions of ASME 117 (1995), 234-242.

[3] C.M. Harris, Shock and Vibration Handbook, New York: McGraw-Hill, 1996.

[4] M.P. Cartmell, Introduction to Linear, Parametric and Nonlinear Vibrations, London: Chapman and Hall, 1990.

[5] A. Tondl, T. Ruijgrok, F. Verhulst and R. Nabergoj, Autoparametric Resonance in Mechanical Systems, Cambridge: Cambridge University Press, 2000.

[6] A.H. Nayfeh and D.T. Mook, Nonlinear Oscillations, New York: John Wiley \& Sons, 1979.

[7] R.S. Haxton and A.D.S. Barr, The autoparametric vibration absorber, Journal of Engineering for Industry 94(1) (1972), 119-124.

[8] H. Hatwal, A.K. Mallik and A. Ghosh, Forced nonlinear oscillations of an autoparametric system - Parts 1 and 2, Journal of Applied Mechanics 50 (1983), 657-662 and 663-668.

[9] M.P. Cartmell and J. Lawson, Performance enhancement of an autoparametric vibration absorber, Journal of Sound and Vibration 177(2) (1994), 173-195.

[10] M.P. Cartmell, On the need for control of nonlinear oscillations in machine system, Meccanica 38 (2003), 185-212.

[11] Y.N. Kyrichko, K.B. Blyuss, A. Gonzalez-Buelga, S.J. Hogan and D.J. Wagg, Real-time dynamic substructuring in a coupled oscillator pendulum system, Proc Royal Society A 462 (2006), 1271-1294.

[12] Y. Song, H. Sato, Y. Iwata and T. Komatsuzaki, The response of a dynamic vibration absorber system with a parametrically excited pendulum, Journal of Sound and Vibration 259(4) (2003), 747-759.

[13] S.R. Hsieh and S.W. Shaw, The stability of modes at rest in a chaotic system, Journal of Sound and Vibration 138(3) (1990), 421-431.

[14] S.J. Zhu, Y.F. Zheng and Y.M. Fu, Analysis of non-linear dynamics of a two-degree-of-freedom vibration system with non-linear damping and non-linear spring, Journal of Sound and Vibration 271(1) (2004), 15-24.

[15] G. Verros and S. Natsiavas, Forcing induced asymmetry on dynamical systems with cubic non-linearities, Journal of Sound and Vibration 233(2) (2000), 279-295. 
[16] X. Jiang, D.M. Macfarland, L.A. Bergman and A.F. Vakakis, Steady state passive nonlinear energy pumping in coupled oscillators: theoretical and experimental results, Nonlinear Dynamics 33 (2003), 87-102.

[17] J. Awrejcewicz and V.A. Krysko, Introduction to Asymptotic Methods, Chapman and Hall, Boca Raton: CRC Press, 2006. 

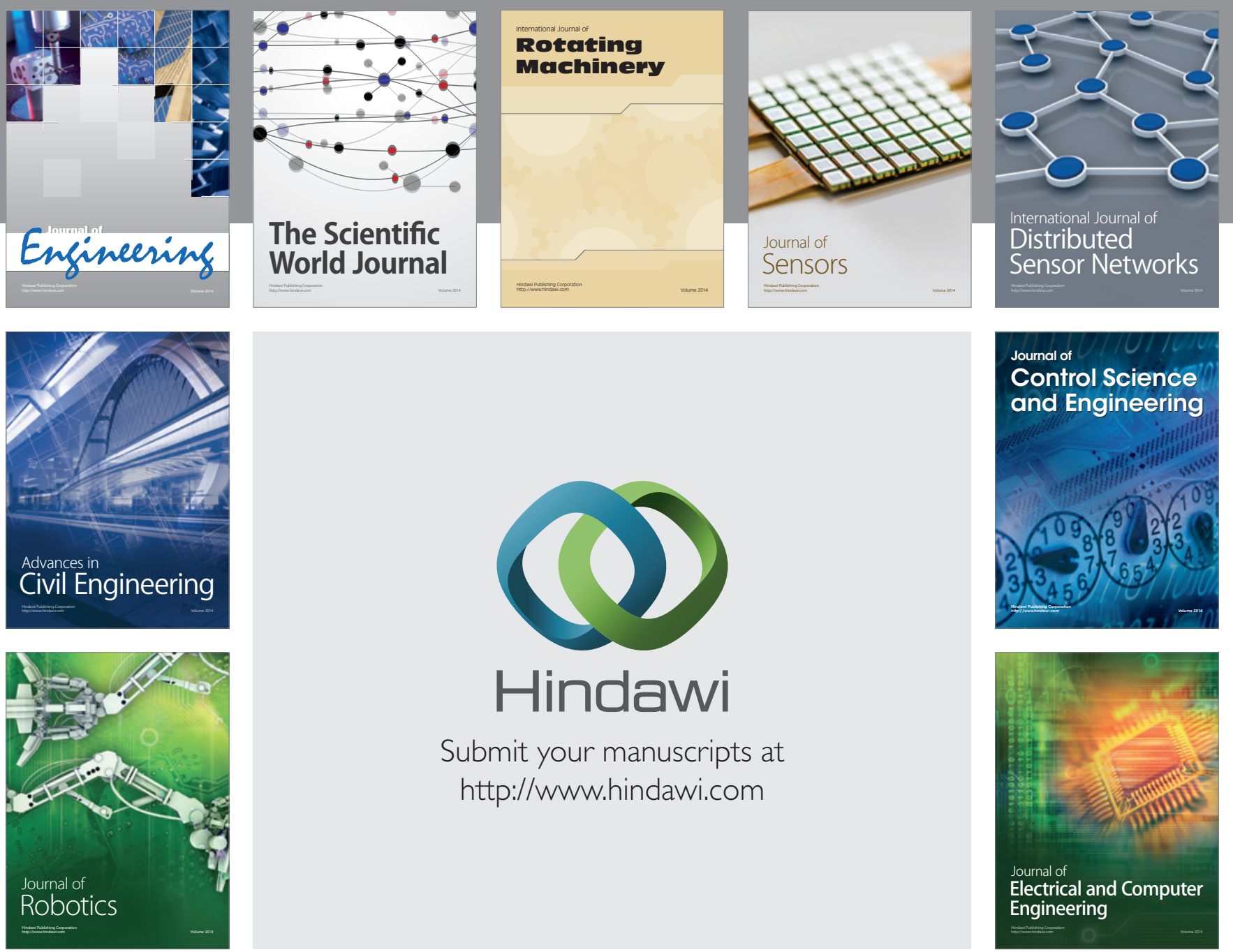

Submit your manuscripts at

http://www.hindawi.com
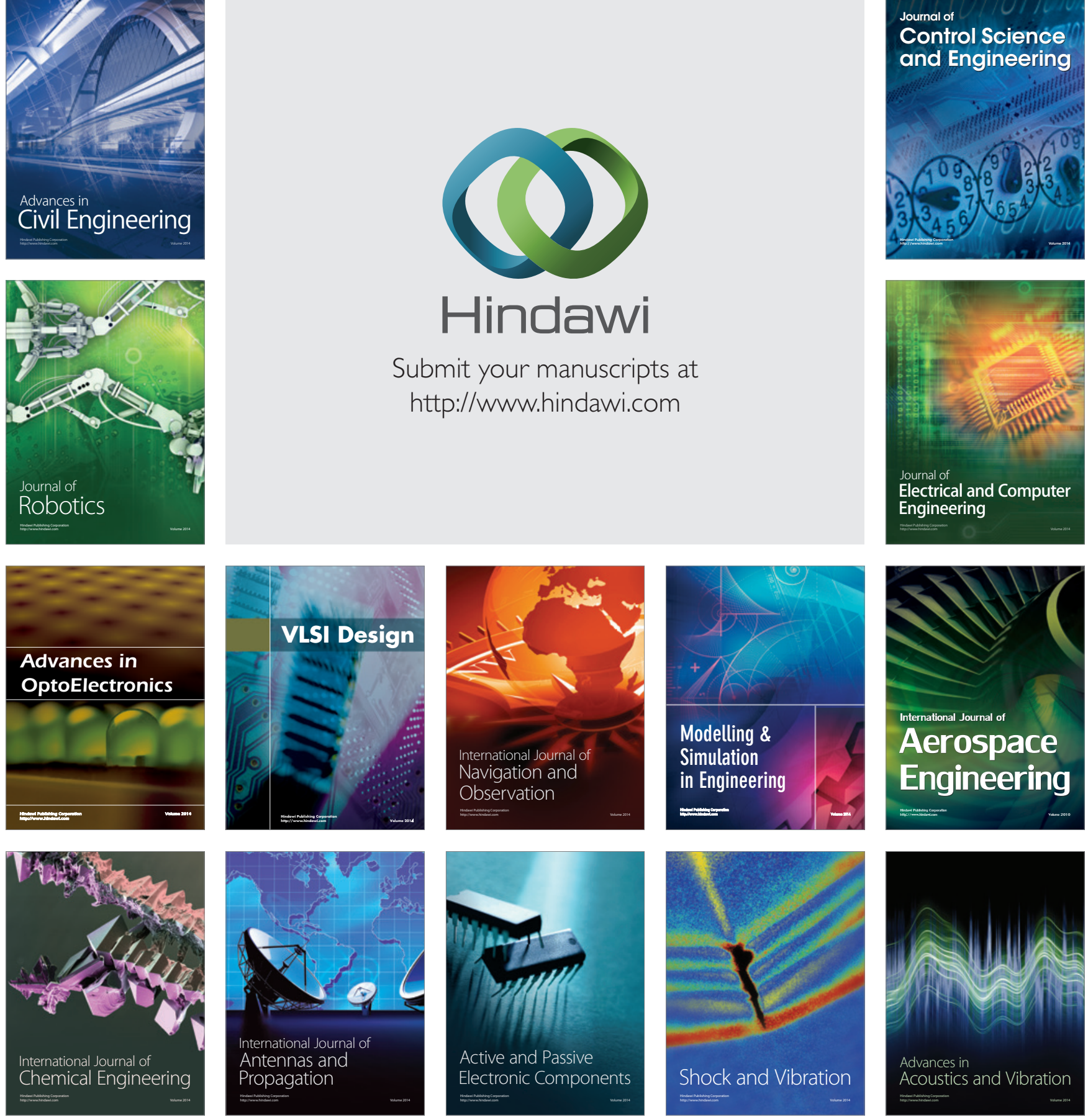